\title{
Mesenchymal stem cells-regulated Treg cells suppress colitis-associated colorectal cancer
}

\author{
Rui-jing Tang ${ }^{1}$, Su-nan Shen ${ }^{1,2}$, Xiao-yin Zhao', Yun-zhong Nie', Yu-jun Xu1', Jing Ren', Ming-ming Lv' \\ Ya-yi Hou ${ }^{1,2^{*}}$ and Ting-ting Wang ${ }^{1,2^{*}}$
}

\begin{abstract}
Introduction: Previous studies have produced controversial results regarding whether mesenchymal stem cells (MSCs) promote or inhibit tumor development. Given the dual role of MSCs in inflammation and cancer, in this study the colitis-associated colorectal cancer (CAC) model was used to examine whether umbilical cord tissue-derived MSCs could prevent neoplasm by inhibiting chronic inflammation.

Methods: MSCs were obtained and identified using flow cytometry. Colitis-associated colorectal cancer model was induced using azoxymethane (AOM) and dextran sulfate sodium (DSS) and MSCs were injected intravenously twice. Levels of immune cells in mesenteric lymph node including regulatory T (Treg) cells were detected using flow cytometry. Naiive T cells and Jurkat cells were co-cultured with MSCs and the effect of MSCs on Treg cells differentiation was evaluated.

Results: After injection through tail vein, MSCs could migrate to colon and suppress colitis-related neoplasm. This tumor suppressive effect was characterized by longer colon length, decreased tumor numbers and decreased expression of Ki-67. Moreover, MSCs alleviated the pathology of inflammation in the colitis stage of CAC model and inhibited inflammation cytokines both in colon and serum. Furthermore, Treg cells were accumulated in mesenteric lymph node of MSCs-treated mice while the percentage of T helper cells 2 (Th2) and Th17 were not changed. Of note, MSCs secreted transforming growth factor- $\beta$ (TGF- $\beta$ ) enhanced the induction of Treg cells from naïve T cells. The conditioned medium of MSCs also activated Smad2 signaling, which has been reported to regulate Treg cells.
\end{abstract}

Conclusions: These results proved that MSCs could migrate to colon tissues and induce the differentiation of Treg cells via Smad2 as so to inhibit the colitis and suppress the development of CAC.

\section{Introduction}

The connection between inflammation and tumor development was noticed after Virchow demonstrated that cancer tended to occur at a site of chronic inflammation [1]. Colorectal cancer which includes hereditary, sporadic and colitis-associated colorectal cancer $(\mathrm{CAC})$ is one of the most common malignancies. More and more evidence shows that chronic inflammation of the colon is an important factor for the progression of colorectal cancer [2]. Patients with inflammatory bowel disease (IBD), such as Crohn's disease and ulcerative colitis, have a higher risk of colorectal cancer development than the healthy population. It is now becoming clear that tumor microenvironment, which is largely orchestrated

\footnotetext{
* Correspondence: yayihou@nju.edu.cn; wangtt@nju.edu.cn

${ }^{1}$ The State Key Laboratory of Pharmaceutical Biotechnology, Division of Immunology, Medical School, Nanjing University, Nanjing 210093, China Full list of author information is available at the end of the article
}

by inflammatory cells, is an indispensable participant in the neoplastic process, including cancer cell proliferation, survival and migration [3]. These insights are fostering new anti-inflammatory therapeutic approaches to cancer [4].

Mesenchymal stem cells (MSCs), which are derived from a variety of tissues and have a fibroblast-like morphology, have the capability of self-renewal and differentiation. MSCs can migrate to the site of tissue damage induced by inflammation and play an antiinflammatory role through regulation of the function of dendritic cells, natural killer cells (NK cells), T cells, and $\mathrm{B}$ cells [5]. MSC can also induce regulatory T (Treg) cells and maintain the capability of Treg cells [6-8]. These properties, which are useful for therapeutic purposes, have recently been found to be abused by cancer cells for their own end. In contrast, reports show that MSCs can inhibit tumor growth under certain circumstances. Our previous 
study has demonstrated that MSCs can alleviate inflammatory disorders in dextran sulfate sodium (DSS)-induced colitis [9]. Given the dual role of MSCs in inflammation and cancers, we hypothesized that MSCs may have an effect on the initiation and progression of CAC.

The role of the immune response in the formation of CAC is complicated. Chronic colitis accompanied by a large accumulation of $\mathrm{T}$ helper cell 1 (Th1), Th2 and Th17 promotes neoplastic risk, whereas excessive immunosuppression regulated by Treg cells enhances the survival of tumor cells $[1,10,11]$. Many researchers have reported that excessive Th1 cells in intestinal mucosa are the main reason for chronic colitis; these cells produce interferon (IFN)- $\gamma$ and interleukin-2 (IL-2) $[12,13]$. Meanwhile, CAC was also characterized as a Th2/Th17 disease accompanied by overproduction of cytokines such as IL-4, IL-5, IL-13 and IL-17 [14,15]. Importantly, Treg cells, which are important in regulating immune responses by selectively suppressing effector $\mathrm{T}$ cells, are believed to play an important role in gut homeostasis and limiting intestinal inflammation [16-18].

Given the dual regulatory effect of MSCs, we hypothesized that MSCs, which modulate immune cells including Treg cells, may have effective anti-inflammation effects on colitis and eventually suppress CAC. To test this hypothesis, MSCs were obtained and injected intravenously in CAC mouse. The therapeutic effects of MSCs on both inflammation and tumor stage of $\mathrm{CAC}$ were investigated.

\section{Methods}

\section{Mice and CAC model}

The CAC model was induced in C57BL/6 male mice (eight weeks of age) purchased from the Model Animal Research Center of Nanjing University. All of the animals received care according to the Guide for the Care and Use of Laboratory Animals. The protocol was approved by the Committee on the Ethics of Animal Experiments of Nanjing University Medical School. Mice were divided into four groups: normal group untreated with MSCs $(\mathrm{n}=12)$; normal group treated with MSCs $(n=12)$; tumor group untreated with MSCs $(\mathrm{n}=16)$; and tumor group treated with MSCs $(\mathrm{n}=16)$. After treatment with intraperitoneal azoxymethane (AOM) $(10 \mathrm{mg} / \mathrm{kg}, 13.4 \mathrm{M}$, purity $\geq 98 \%$; SIGMA, Aldrich, St. Louis MO, USA), three cycles of $2 \%(\mathrm{w} / \mathrm{v})$ DSS (40,000 Da; SIGMA, Aldrich, St. Louis MO, USA) in the drinking water (7 days DSS and 14 days water) induced long-lasting chronic DSS colitis in these mice, as previously described [19]. Mice were checked daily for behavior. At day 5 and day 26, mice were intravenously injected with $2 \times 10^{5}$ MSCs diluted in $200 \mu \mathrm{l}$ phosphatebuffered saline (PBS) or a vehicle control (PBS alone) through the tail vein. The mortality rate of this animal model is $0 \%$ to $5 \%$. Half of the mice in each group were sacrificed at day 33 to investigate the progression of inflammation, and the others were sacrificed at day 70 to evaluate the development of tumor. The degree of colitis was evaluated daily by scoring the clinical disease activity (0 to 4), which includes stool status, fecal blood and weight loss. Colons removed from cecum to anus were collected, photos were taken, and the colons prepared for histopathological analysis, immunohistochemistry and quantitative real-time PCR (Q-PCR) analysis.

\section{Preparation of umbilical cord-derived MSCs}

Human umbilical cords were obtained from the Affiliated Drum Tower Hospital of Nanjing University Medical School. The Drum Tower Hospital ethics committee approved the consent forms and the protocol for evaluation of the tissue and patients gave written consent. The umbilical cords were rinsed with PBS in penicillin and streptomycin, and then the umbilical arteries and veins were removed. The remaining tissue was cut into 1 to $2 \mathrm{~mm}$ pieces and floated in (Dulbecco's) modified Eagle's medium/nutrient F-12 Ham's (Gibco, Grand Island, NY, USA). The pieces were subsequently digested in an enzyme cocktail (hyaluronidase $5 \mathrm{U} / \mathrm{ml}$, collagenase $125 \mathrm{U} / \mathrm{ml}$, and dispase $50 \mathrm{U} / \mathrm{ml}$; SIGMA) for three hours at $37^{\circ} \mathrm{C}$. After this tissue was crushed with forceps and large pieces were removed, human umbilical cord MSCs were harvested and plated into a culture flask. The cells were incubated at $37^{\circ} \mathrm{C}$ in an incubator with $5 \% \mathrm{CO}_{2}$ at saturating humidity. When cells developed colonies and reached $70 \%$ to $80 \%$ confluence, the cells were detached with $0.25 \%$ trypsin-ethylenediaminetetraacetic acid (EDTA) and passaged to a culture flask for further expansion.

\section{Determination of MSC migration}

MSCs migrating to the intestines in vivo were detected by labeling with the fluorescent dye CM-DiI (Molecular Probes Eugene, Oregon, USA). Before being injected, MSCs were resuspended in PBS containing $2 \mu \mathrm{g}$ CM-DiI, and then incubated at $37^{\circ} \mathrm{C}$ for five minutes followed by fifteen minutes at $4^{\circ} \mathrm{C}$ in the dark. Cells were washed twice with PBS and resuspended to be injected in the tail vein. Mice were killed (at day 8 and day 12) and tissues were collected to make paraffin sections. MSCs were determined by fluorescence microscope detection of CM-DiI dye (red).

\section{Histopathological analysis}

For histopathological examination, intestinal tissue was embedded in paraffin, sectioned and stained with hematoxylin and eosin (H \& E). Inflammation was blindly scored from 0 to 4 as follows: 0 , no signs of inflammation; 1 , low leukocyte infiltration; 2, moderate leukocyte infiltration; 3, high leukocyte infiltration, moderate fibrosis, high vascular density, thickening of the colon wall, moderate goblet cell loss, and focal loss of crypts; and 4, transmural infiltration, massive loss of goblet cells, extensive fibrosis, and diffuse loss of crypts. 


\section{Immunohistochemistry}

Paraffin-embedded intestinal sections were mounted on slides, dewaxed in xylene, and rehydrated in graded alcohol washes. Slides were heated by microwave in $0.01 \mathrm{~mol} / \mathrm{L}$ trisodium citrate buffer for antigen retrieval. Bovine serum albumin, $5 \%$, (BSA) was used to block nonspecific bonding sites for 30 minutes and $3 \% \mathrm{H}_{2} \mathrm{O}_{2}$ was used to suppress endogenous peroxidase activity. Slides were then incubated with primary antibody (Ki-67, p-P65 and FoxP3) overnight at $4^{\circ} \mathrm{C}$ and washed with Tris-buffered saline (TBS) before incubation with labeled polymer horseradish peroxidase rabbit antibody for 30 minutes. Counterstaining was performed with hematoxylin. Slides were dehydrated through ascending alcohols to xylene and mounted to take photos. The proliferation index of Ki-67 and p-P65 was given as the ratio between positive nuclei and total number of nuclei per crypt. Five visual fields per sample were selected randomly to analyze at least 20 crypts.

\section{mRNA isolation and quantitative-PCR}

Total RNA was extracted from intestinal tissue with TRIzol Reagent (Invitrogen, Carlsbad, CA, USA) according to the manufacturer's instructions. A total of $1 \mu \mathrm{g}$ RNA was used as the template for single strand cDNA synthesis. Quantitative PCR (Q-PCR) for GAPDH, IL- $1 \beta$, IL-6 and TNF- $\alpha$ was performed on an Applied Biosystems 7300 Sequence Detection System (Applied Biosystems, Foster city CA, USA) using SYBR green dye (Invitrogen, USA), programmed for $95^{\circ} \mathrm{C}$ for 10 minutes, then 40 cycles of $95^{\circ}$ $\mathrm{C}$ for 15 seconds, $60^{\circ} \mathrm{C}$ for 30 seconds, and $72^{\circ} \mathrm{C}$ for 30 seconds. All reactions were run in triplicate.

\section{Cytokine analysis by Bio-Plex Assay}

IL- $\alpha$, IL- $\beta$, IL-3, IL-4, IL-5, IL-6, IL-12, IL-13, G-CSF and RANTES in sera were quantitated to investigate the change in the immune system using the 10-plex Bio-Rad (Bio-Rad, Hercules CA, USA), according to the manufacturer's instructions.

\section{T cell preparation and treatment}

CD4 ${ }^{+} \mathrm{CD}_{2} \mathrm{~L}^{+} \mathrm{T}$ cells were positively selected from murine splenocytes using a magnetic-activated cell-sorting system (Miltenyi Biotec, Bergisch Gladbach Germany). High purity naive $\mathrm{T}$ cells were plated into 12-well culture plates and cultured in Roswell Park Memorial Institute (RPMI) 1640 (Gibco, Grand Island, NY, USA) containing $10 \%$ fetal bovine serum (FBS) and ant-CD3/ CD28 (eBioscience, San Diego, CA, USA) monoclonal antibodies. Then MSCs were added in the top chamber of 12 -well transwell chambers with $3-\mu \mathrm{m}$ pore polycarbonate filters and co-cultured with naive T cells at $37^{\circ} \mathrm{C}$ in an incubator with $5 \% \mathrm{CO}_{2}$ at saturating humidity. Transforming growth factor- $\beta 1$ (TGF- $\beta 1$ ) (Miltenyi Biotec Bergisch Gladbach, Germany) and anti-TGF- $\beta 1$ antibody (ab64715, Abcam, USA) was also added in the same chamber to evaluate the effect of MSCs on the differentiation of naive $\mathrm{T}$ cells. Overall, we had four groups: $\mathrm{CD} 4^{+} \mathrm{T}$ cells alone, $\mathrm{CD} 4^{+} \mathrm{T}$ cells cultured with TGF- $\beta, \mathrm{CD}^{+}{ }^{+} \mathrm{T}$ cells cultured with MSCs, and $\mathrm{CD} 4^{+} \mathrm{T}$ cells cultured with anti-TGF- $\beta$ /MSCs. Cells at the bottom of the chamber were collected and analyzed.

\section{Flow cytometric analysis}

Cells were stained with CD29, CD44, CD73, CD90, CD105, HLA-ABC, CD14, CD31, CD34, CD45, HLADR, CD3, CD4, CD8, CD25, IL-4, IL-17, and FoxP3 (eBioscience). In addition, intracellular IL-4, IL-17 and FoxP3 staining were treated with a FoxP3 staining buffer set (eBioscience). Flow cytometry was carried out on the FACScalibur flow cytometer (BD, San Diego, USA). Data were analyzed using FlowJo software (Treestar, Inc., San Carlos, CA, USA).

\section{Western blots}

Jurkat cells, which were stimulated by MSCs conditioned medium, were lysed in a buffer containing 50 $\mathrm{mM}$ Tris- $\mathrm{Cl} \mathrm{pH}$ 8.0, $150 \mathrm{mM} \mathrm{NaCl}, 0.02 \% \mathrm{NaN} 3,0.1 \%$ SDS, $100 \mathrm{mg} / \mathrm{ml}$ phenyl-methylsufonyl fluoride (PMSF), $1 \mathrm{mg} / \mathrm{ml}$ aprotinin, $1 \%$ Triton. A total of $10 \mu \mathrm{g} / \mathrm{mL}$ aprotinin, $10 \mu \mathrm{g} / \mathrm{mL}$ leupeptin, $1 \mathrm{mM}$ dithiothreitol, 1 $\mathrm{mM}$ paranitrophenyl phosphate, and $0.1 \mathrm{mM} \mathrm{Na}_{3} \mathrm{VO}_{4}$ were added as protease and phosphatase inhibitors. After centrifugation, protein samples were subjected to $10 \%$ SDS-PAGE and transferred onto polyvinylidene difluoride (PVDF) membranes (Roche, Mannheim, Germany). The membranes were blocked in TBST (1 $\mathrm{mM}$ Tris-HCl, pH 7.4, $150 \mathrm{mM} \mathrm{NaCl}, 0.05 \%$ Tween-20) containing 5\% BSA for 0.5 hour and subsequently incubated overnight at $4^{\circ} \mathrm{C}$ with diluted primary antibodies against GAPDH, Smad2 and Phospho-Smad2. The band was quantified using the FluorChem FC2 system (Alpha Innotech Corporation St. Leonardo, CA, USA).

\section{Statistical analysis}

Results are presented as mean \pm standard error of the mean (SEM). Student's t-test was used to compare between two groups. One-way analysis of variance (ANOVA) was used to compare among three or more groups. A $P$ value of $<0.05$ was considered significant, and survival curves were performed with GraphPad Prism software (San Diego, CA, USA).

\section{Results}

MSCs migrate to the colon and decrease the incidence of colitis-related neoplasm

MSCs were isolated from human umbilical cord tissue and confirmed by analyzing MSC-related cell surface 
antigens using flow cytometry. As shown in Figure 1A, MSCs were successfully obtained with positive markers of CD29, CD44, CD73, CD90, CD105 and HLA-ABC, and with negative markers of CD14, CD31, CD34, CD45 and HLA-DR. We then detected the migration activity of MSCs in mice through tail vein injection. Compared with liver, more accumulation of MSCs was found in lung for three days after injection in normal mice. In the tumor group, that is, the mice treated with AOM and $2 \%$ DSS, the amount of MSCs in lung was decreased (Figure 1B). At the same time, MSCs were also detected in colon (Figure 1C). These results indicated that MSCs were sufficiently dynamic to migrate in vivo and could migrate to colon tissues when the intestinal epithelia of mice were damaged by DSS. At day 12, MSCs still were found in the colon tissue (Figure 1D), suggesting that this time interval provided a potential therapeutic chance of MSCs in colon tissues.
The CAC model was successfully induced by injecting mice with AOM and 2\% DSS (Figure 2A). Mice in the tumor group developed colon tumors mainly in the distal to middle colon, which is the predominant localization of human colorectal tumors. We noticed that mice treated with MSCs had fewer macroscopic tumors (Figure 2B and C) and longer colon length (Figures $2 \mathrm{E}$ ). The average size of the tumor was not affected in the two groups (Figure 2D). Moreover, immunohistochemical analysis showed that Ki-67 was lower in colon tissues of mice treated with MSCs than in those without treatment (Figure 2F and G). These data confirmed that MSCs could inhibit tumor development in the CAC model.

MSCs alleviate the pathology of DSS-induced chronic colitis To induce colorectal cancer successfully, the chronic colitis should emerge first in three cycle inductions. It is now commonly accepted that inflammation contributes

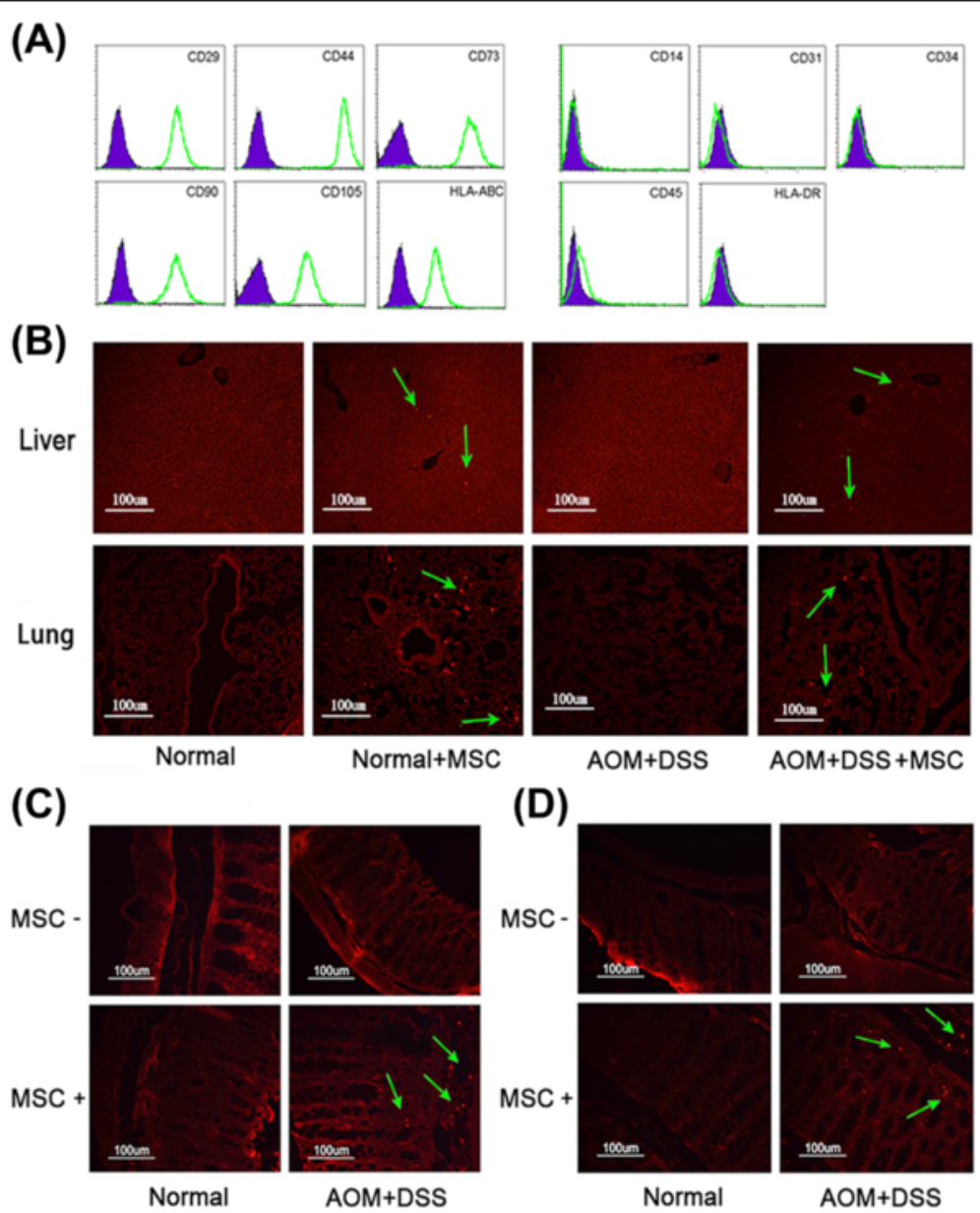

Figure 1 The phenotype and migration pathway of MSCs in the DSS/AOM-induced model. (A) Expression of MSC phenotype was detected by FACScalibur flow cytometer. (B) Liver and lung in each group were moved, collected and paraffin sections made at day 6 to detect the migration pathway of MSCs stained with CM-Dil (red). (C) and (D) show colon tissue collected to detect the migration pathway of MSCs at day 6 and day 10. AOM, azomethane; DSS, dextran sulfate sodium; FACS, fluorescence activated cell sorting; MSCs, mesenchymal stem cells. 


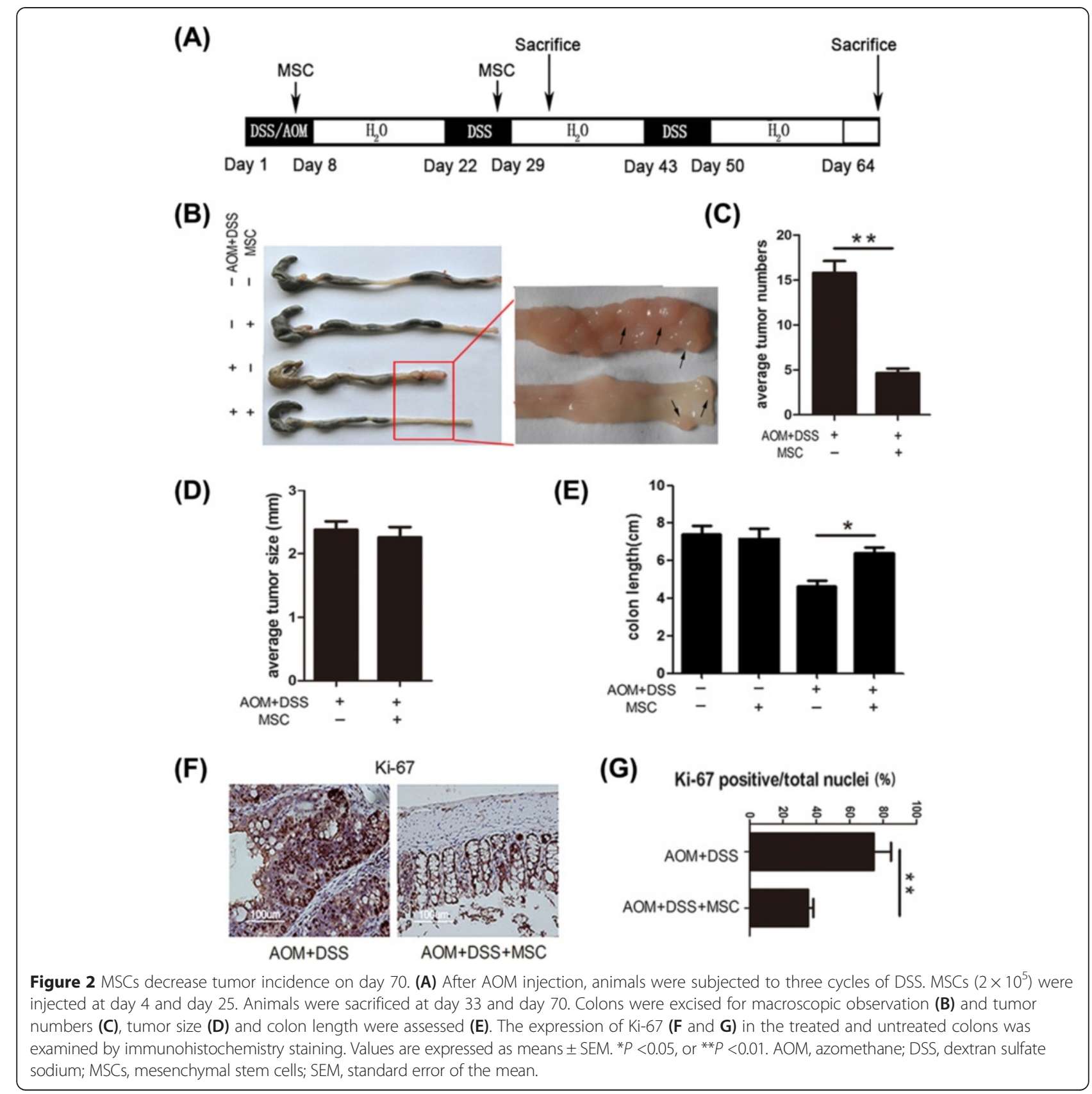

to the initiation, promotion, and progression of tumor development. To confirm that MSCs inhibit carcinogenesis through inhibition of colonic inflammation, half of the mice in the tumor group were sacrificed on day 32 . As shown in Figures 3A to D, MSC treatment significantly alleviated chronic inflammation with improved clinical scores. MSC treatment also improved colitis characteristics with increased body weight $(P<0.05)$ and colon length $(P<0.05)$. Moreover, histological examination of the colon in mice without MSCs showed patchy ulceration, epithelial cell loss, reduction of the density of tubular glands, focal loss of crypts, inflammatory cell infiltrates and transmural inflammation involving all layers of the bowel wall. Expectedly, these colitis characteristics were reversed after MSC treatment (Figures $3 \mathrm{E}$ and $F, P<0.05)$. These results consistently demonstrated that during the development of CAC, MSCs had a protective function on DSS-induced colitis by inhibiting excessive inflammation in colon tissues.

MSCs inhibit inflammatory cytokines in colon and serum Cytokine levels are critical evidence to show the effect of immune cells on CAC growth. In order to test the effect of MSCs on levels of inflammatory cytokines, serum 
(A)

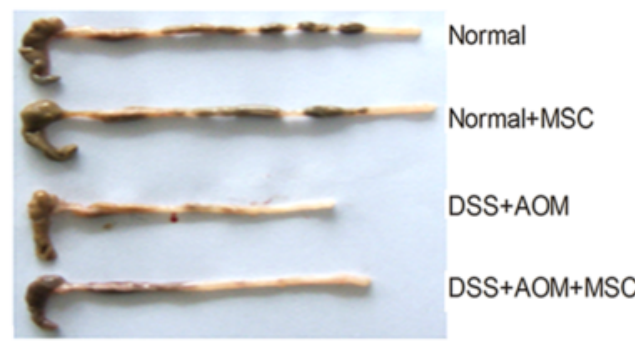

(C)

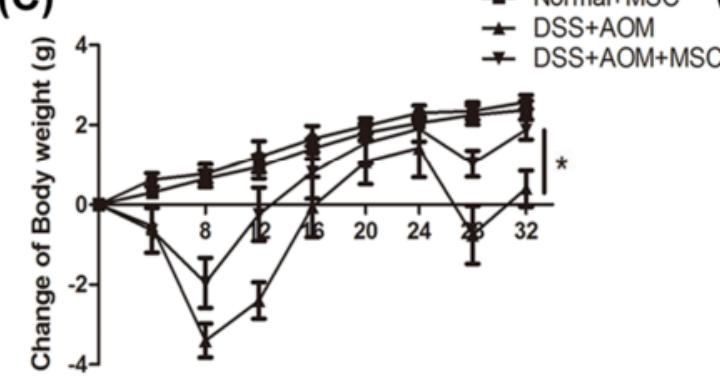

(E)

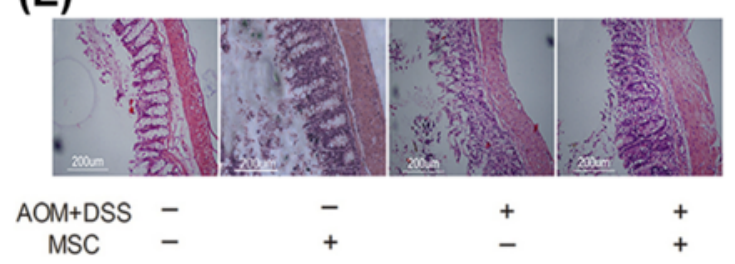

(B)

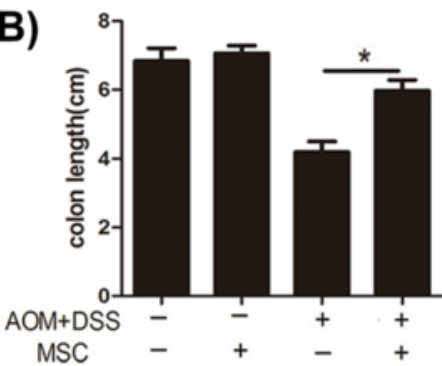

(D)

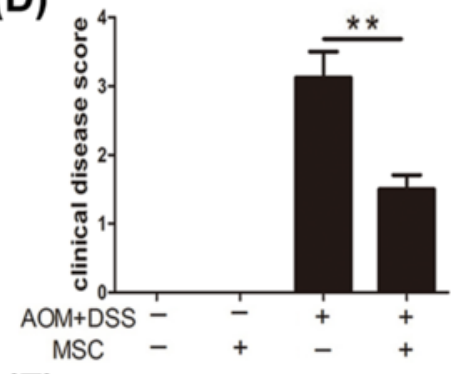

(F)

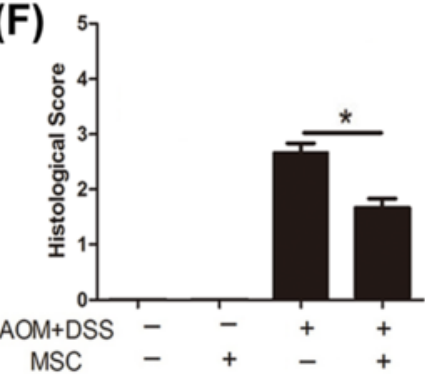

Figure 3 MSCs protect mice against the development of DSS-induced colitis on day 33. Colons were excised for macroscopic observation (A) and assessment of colonic length (B). (C) The changes in body weight were calculated based on initial body weight. (D) The clinical disease score of colitis, which includes stool status, fecal blood, length of colon and weight loss, was used to evaluate the therapeutic effect of MSCs. (E) Colon sections from mice with different treatments were examined using $\mathrm{H} \& \mathrm{E}$ staining. (F) Histology scores were derived from microscopic analysis of longitudinal colon sections from each mouse. Values are expressed as means \pm SEM. ${ }^{*} P<0.05$, or ${ }^{* *} P<0.01$. DSS, dextran sulfate sodium; MSCS mesenchymal stem cells; SEM, standard error of the mean.

from different groups of mice was collected and cytokine levels were detected using a Bio-Plex cytokine kit. Compared with the untreated group, inflammatory cytokines, including IL-1 $\alpha$, IL-1 $\beta$, IL-5, IL-6 and IL-12, were decreased significantly after treatment with MSCs (Figure 4A, $P<0.05$ ).

Many DNA-binding proteins are aberrantly activated in response to inflammatory stimuli, which can cause inappropriate induction of various proinflammatory genes in tumor cells, tumor-associated stromal cells and surrounding host tissues. NF- $\mathrm{kB}$, one of these DNA-binding proteins, has been identified as a potential molecular bridge between inflammation and cancer, and can induce proinflammatory cytokines such as IL-1, IL-6 and TNF$\alpha$. mRNA in colon was extracted and gene expressions of IL-1 $\beta$, IL- 6 and TNF- $\alpha$ were examined. As shown in Figure $4 \mathrm{~B}$, the expressions of IL- $1 \beta$, IL- 6 and TNF- $\alpha$ were reduced $(P<0.05)$ after treatment with MSCs.
Immunohistochemical analysis also showed that the protein level of p-P65 was decreased in colon tissues treated with MSCs, compared with colon tissues without treatment (Figure 4C).

\section{Treg cells are up-regulated in the progression of chronic colitis}

To detect the regulatory effect of MSCs on immune cells, flow cytometry was used to analyze changes of adaptive immune cells in mesenteric lymph nodes after treatment with MSCs for 33 days (colitis stage). As shown in Figure $5 \mathrm{~A}$, the mean percentage of $\mathrm{CD} 4^{+} \mathrm{T}$ cells and $\mathrm{CD} 8^{+} \mathrm{T}$ cells reflected no difference among groups as well as Th2 and Th17 cells. As is well known, forkhead box P3 (FoxP3) is the transcription factor of Treg cells. Notably, CD $4^{+} \mathrm{CD} 25^{+} \mathrm{FoxP}^{+}$Treg cells were significantly up-regulated by MSC treatment (Figure 5B, $P<0.05)$. These phenomena suggest that MSC may just 


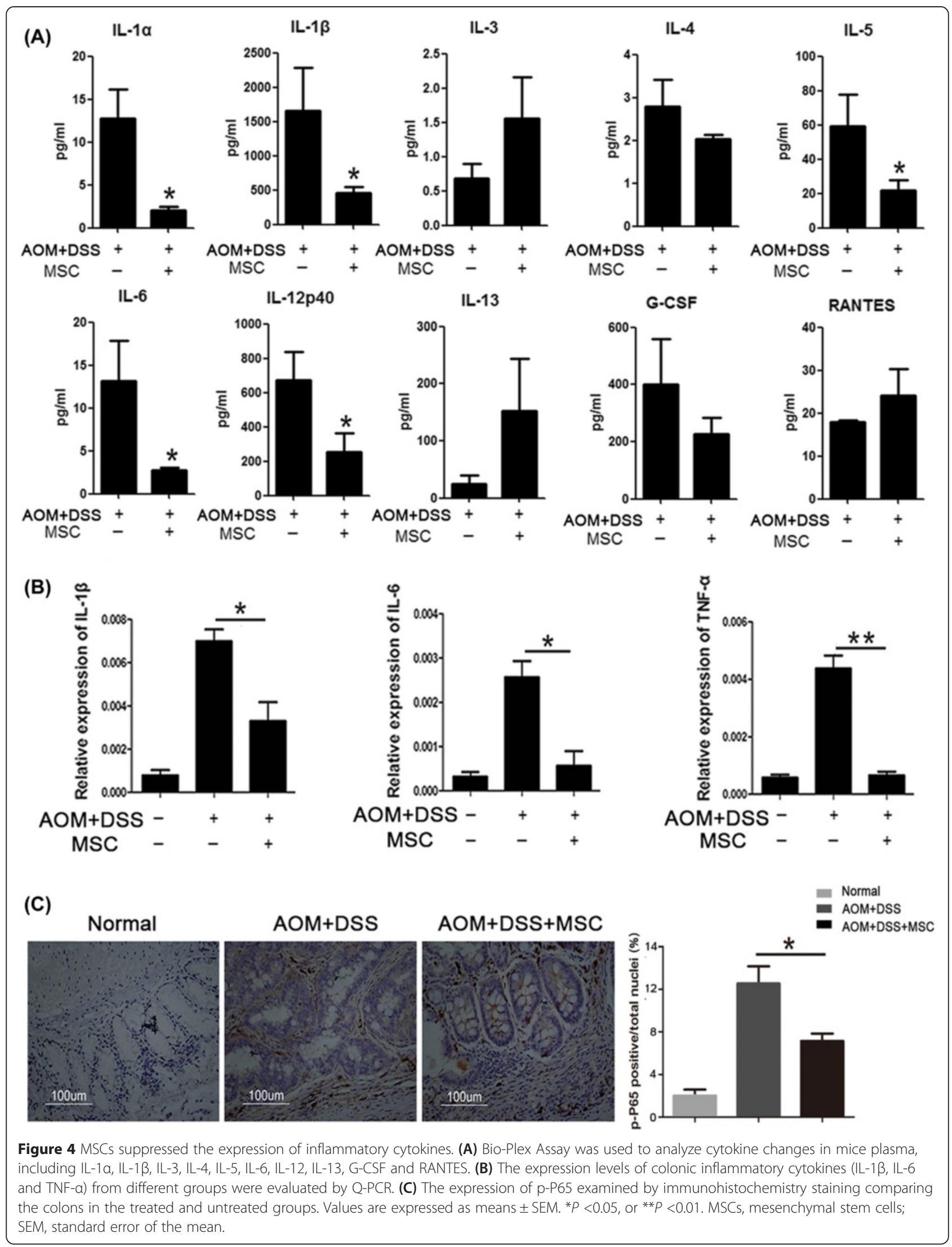


(A)
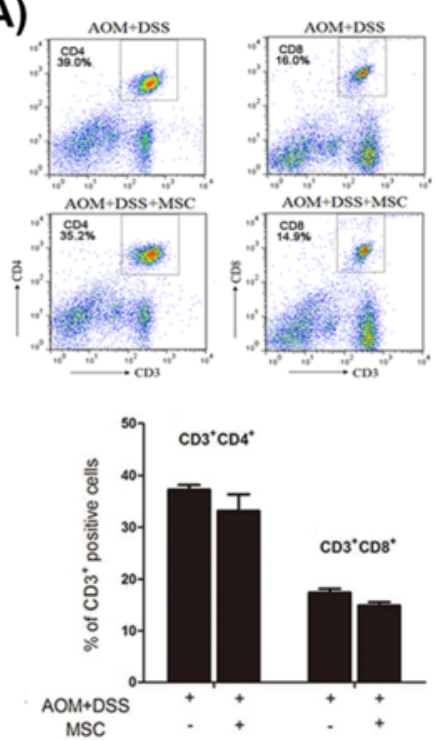

(C)

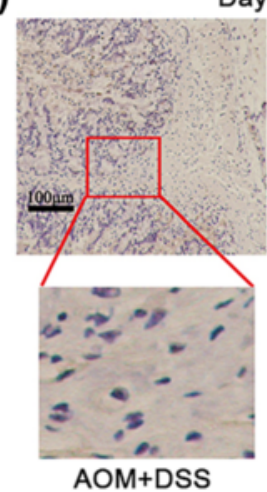

Day 33

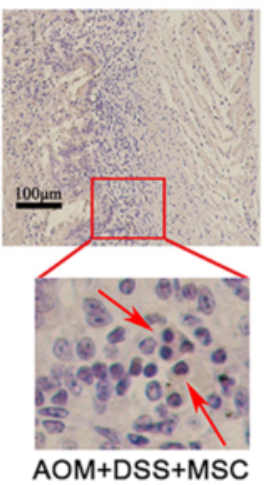

(B)
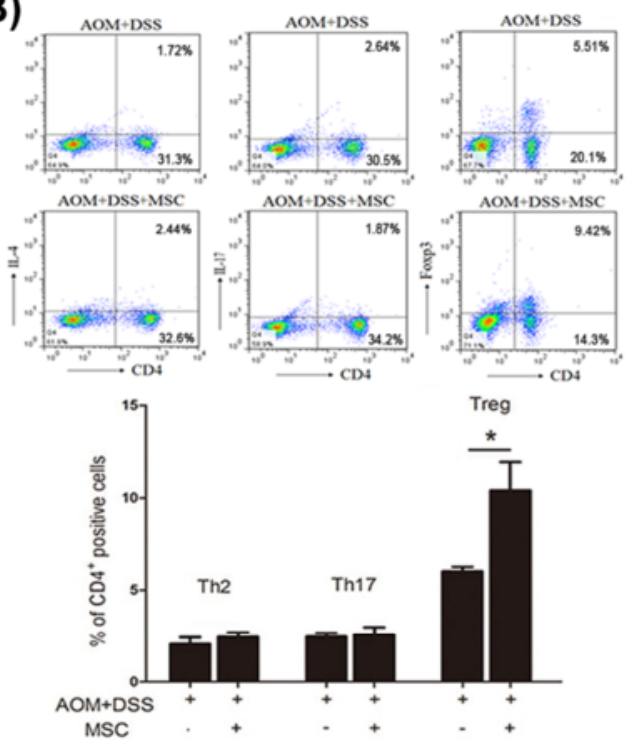

(D)

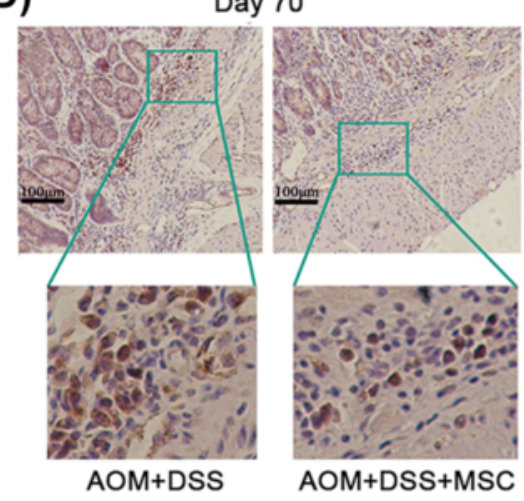

Figure $\mathbf{5}$ The effect of MSCs on the accumulation of $\mathrm{CD} 4^{+} \mathrm{CD} 25^{+} \mathrm{FoxP}^{+}$Treg cells. (A) Flow cytometry analysis of the mean percentage of $\mathrm{CD} 4^{+} \mathrm{T}$ cells and CD8 ${ }^{+} T$ cells in mesenteric lymph. (B) Flow cytometry analyzed the mean percentage of Th2, Th17 and Treg cells in mesenteric lymph. Values are expressed as means \pm SEM. ${ }^{*} P<0.05$. Colons collected at day 33 (C) and day 70 (D) stained with Foxp3 examined by immunohistochemistry. MSCs, mesenchymal stem cells; SEM, standard error of the mean; Treg cells, regulatory $T$ cells.

induce the accumulation of Treg cells in mesenteric lymph nodes to suppress excessive inflammation after MSCs migrate to the colon. To further confirm the involvement of Treg cells in intestinal inflammation, FoxP3 stained by immunohistochemistry was used to analyze whether Treg cells infiltrated to the stroma and epithelia of the intestine. Interestingly, according to the density of FoxP3, we found that Treg cells infiltrated in intestinal stroma more in the MSC treated group than in the untreated group (Figure $5 \mathrm{C}$ ). We also determined the density of Treg cells at day 70 (tumor stage). In contrast, the results showed that the infiltrated Treg cells were found less in the MSCs group compared to the neoplasm in the untreated group (Figure 5D). Therefore, these results indicated that MSCs indeed were involved in inducing Treg cells to suppress colitis.

\section{MSCs increase Foxp3+ cells via activating Smad2} signaling in vitro

To explore how MSCs regulate Treg cells, naive $\mathrm{CD} 4^{+} \mathrm{T}$ cells were separated from mouse spleen and then stimulated by anti-CD3/CD28 to maintain survival in vitro. After co-culture with MSCs for two days, the expression of FoxP3 slightly changed (Figure 6A). However, after three days, the $\mathrm{FoxP}^{+}$cells were increased to $13.7 \%$ in the TGF- $\beta$ alone group and $20.2 \%$ in the MSC group. To prove that the induction effect of MSCs is through TGF$\beta$, anti-TGF- $\beta$ was added to the MSC supernatant. The percentage of $\mathrm{FoxP}^{+}$cells returned to its normal level in the anti-TGF- $\beta$ group. Moreover, the MSC conditioned medium was then used to stimulate Jurkat cells. Mechanically, phosphorylation-Smad2 was activated after 30 minutes treatment with MSCs while JNK and p38 signaling showed no change (Figure $6 \mathrm{~B}$ and $\mathrm{C}$ ). 
(A)
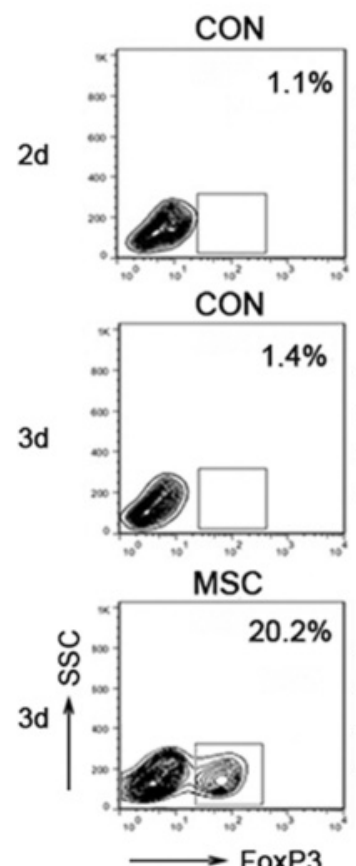

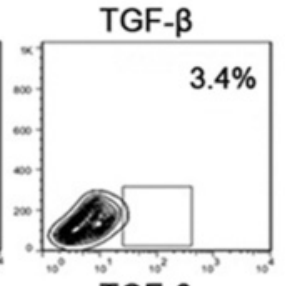

TGF- $\beta$

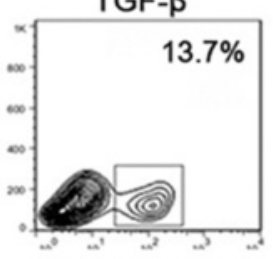

$M S C+a n t i T G F-\beta$

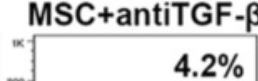

Figure 6 MSCs increase the expression of FoxP3 in naive CD4 ${ }^{+} T$ cells. (A) Naive CD4 ${ }^{+} T$ cells co-cultured with MSCs (ratio 1:5)/TGF- $\beta$ (5 ng/ml) or without MSCs/TGF- $\beta$ in the presence of anti-CD3/CD28 (2 $\mu \mathrm{g} / \mathrm{ml}$ each). T cells were harvested from different treatment groups to assess Foxp3 expression by flow cytometry. (B) MSCs conditioned medium was collected after 48 hours and 72 hours, and then used to stimulate Jurkat cells for different times. were harvested to examine TGF- $\beta$ signaling by Western blotting of Smad2 and phospho-Smad2. TCR signaling was examined by Western blotting of JNK, phospho-JNK, p38 and phospho-p38. (C) The protein level of p-Smad2 was analyzed using Quantity One. Values are expressed as means \pm SEM. ${ }^{*} P<0.05$ or ${ }^{*} P<0.01$. MSCs, mesenchymal stem cells; SEM, standard error of the mean; TCR, T cell receptor; TGF- $\beta$,

transforming growth factor- $\beta$.

These data suggest that MSCs secreting TGF- $\beta$ increased the FoxP3 ${ }^{+}$cell populations via activating phosphorylation of Smad2.

\section{Discussion}

As it is difficult to cure tumors directly, many reports have focused on anti-inflammation to inhibit the initiation and progression of tumors [20]. Recently, some studies have indicated that paeonol [20], glucosamine [21], and digitoflavone [22] can significantly attenuate inflammation and produce proinflammatory cytokines to ameliorate CAC. Our previous research showed that MSCs can regulate immunological balance [23-25] and ameliorate DSS-induced acute colitis [9]. Based on these studies, we designed this experiment to research whether MSCs may inhibit tumorigenesis in the CAC mice model. Our results found that MSCs could migrate to the colon and suppress colitis-related neoplasms. This tumor suppressive effect was characteristized by longer colon length, decreased tumor numbers and decreased expression of Ki-67. Furthermore, MSCs alleviated the pathology of inflammation in the colitis stage of the CAC model. Mechanically, Treg cells were accumulated
(B)

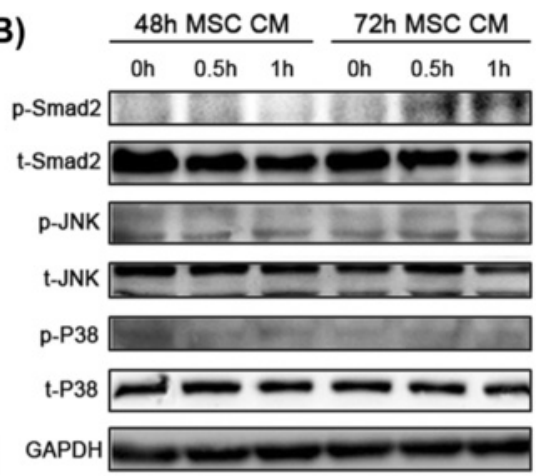

(C)

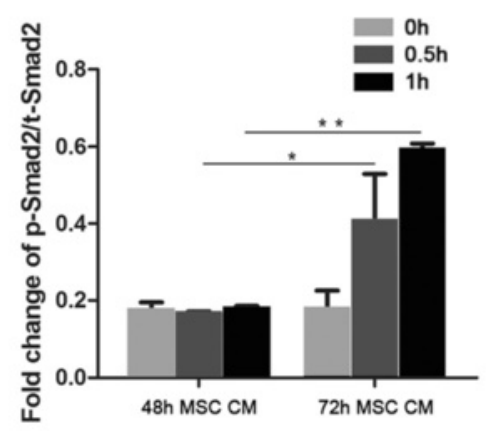

in mesenteric lymph node of MSC-treated mice. In vitro, MSCs secreting TGF- $\beta$ enhanced the induction of Treg cells from naïve $\mathrm{T}$ cells.

The effect of MSCs on cancers is controversial. Many studies have shown that MSCs can promote tumor growth. B16 melanoma cells cannot form tumors in allogeneic mice unless co-injected with MSCs [26]. When human breast cancer cells mixed with bone marrowderived human MSCs were injected in a mouse model, there was a higher speed of metastases [27]. On the other hand, there are also many reports proving that MSCs can inhibit tumor growth. MSCs can exert an anti-tumorigenic effect that is mediated by inhibition of Akt activity in a model of Kaposi's sarcoma [28]. MSCs can also inhibit human liver cancer cell lines and the development of a hepatoma model involving the wnt signaling pathway [29]. However, there is much debate about the function of MSCs in tumor development, and it is difficult to research how MSCs suppress tumors directly. In our CAC model, we found that MSCs could suppress colitis at the colitis stage and eventually suppress tumor development at the tumor stage. Furthermore, inflammatory mediators were detected both in 
blood and intestinal tissues. IL-1 $\beta$, IL- 6 and TGF- $\beta$, which had high levels in colitis [30], were significantly decreased in the MSC treated group. These results together suggest that MSCs can play a therapeutic role in the progression of CAC.

It is believed that Treg cells play an important role in the regulation of colitis. Colitis induced by transferring $\mathrm{CD} 4{ }^{+} \mathrm{CD} 25 \mathrm{RB}^{\text {high }} \mathrm{T}$ cells into $\mathrm{RAG}^{-/-}$mice can be prevented by $\mathrm{CD} 4^{+} \mathrm{CD} 25^{+}$Treg cells [31]. Modulating the balance between Treg and Th17 cells by IL-22 can also reduce the pathological degree in experimental colitis [32]. Here, we found that at the colitis stage, both the colon and mesenteric lymph node had more infiltration of Treg cells in MSC treated group than in the untreated group. On the other hand, our results found that at the tumor stage fewer Treg cells were infiltrated in the MSCs treated group compared with the untreated group. Here, we just injected MSCs twice to suppress inflammation development at the colitis stage, so at the tumor stage whether Treg cell infiltration could be affected by MSCs needs further research.

FoxP3, which has been identified as the transcription factor, is responsible for determining the function of Treg cells. Various signals that induce the expression of FOXP3 have been reported, such as T-cell receptor (TCR), co-stimulatory molecules and cytokine receptors [33]. IL-2 [34] and TGF- $\beta$ [35] are essential cytokines for the differentiation of CD4 + CD25 + FoxP3 + Treg cells from naive $\mathrm{CD} 4+\mathrm{T}$ cells. It has been reported that MSCs can secrete TGF- $\beta$ to interact with NK cells [36]. In vitro, we found that Treg cells were induced when naive CD4 + T cells were co-cultured with MSCs and then the TGF- $\beta$-Smad2 signal was also activated. All these results support our hypothesis that MSCs may secrete TGF- $\beta$ which was quantified in our previous study [24] to induce Treg cells, and then suppress the progression of inflammation-dysplasia-carcinoma in CAC.

\section{Conclusions}

To sum up, our present research analyzed the function of MSCs in CAC. We found that MSCs can inhibit colitis at the colitis stage and eventually suppress the development of colon cancer at the tumor stage. The expressions of inflammatory cytokines in blood and colon tissue were all inhibited by MSCs. Importantly, Treg cells accumulated in colon tissues following MSC treatment. MSCs can raise the expression of FoxP3 in naive CD $4+\mathrm{T}$ cells and activate the TGF- $\beta$-Smad 2 signal. Taken together, our results prove that MSCs can induce Treg cells to suppress chronic inflammation and then suppress the development of CAC.

\section{Abbreviations}

AOM: azoxymethane; BSA: bovine serum albumin; CAC: colitis-associated colorectal cancer; DSS: dextran sulfate sodium; FoxP3: forkhead box P3;
hUCMSCs: human umbilical cord tissue-derived mesenchymal stem cells; IFN: interferon; IL: interleukin; PBS: phosphate-buffered saline; Q-PCR: quantitative PCR; TGF- $\beta$ : transforming growth factor- $\beta$; TNF: tumor necrosis factor; Treg cells: regulatory $T$ cells.

\section{Competing interests}

The authors declare that they have no competing interests.

\section{Authors' contributions}

TRJ: experimental design, data collection and manuscript drafting; SSN: experiment design, acquisition of funding, manuscript drafting and revision; ZXY: partial data collection and data analysis; NYZ: experimental design and data collection; XYJ: material support and data analysis; RJ: material support and data analysis; LMM: material support and data analysis; HYY: experiment design, acquisition of funding, manuscript drafting and revision; WTT: experimental design and final approval of manuscript. All authors read and approved the final manuscript.

\section{Acknowledgements}

This work was supported by National Natural Science Foundation of China (number: 31370899) and Department of Clinical Medicine in Jiangsu Province Science and Technology Projects (project number: BL2014069).

\section{Author details}

${ }^{1}$ The State Key Laboratory of Pharmaceutical Biotechnology, Division of Immunology, Medical School, Nanjing University, Nanjing 210093, China.

${ }^{2}$ Jiangsu Key Laboratory of Molecular Medicine, Nanjing 210093, China.

Received: 22 August 2014 Revised: 30 August 2014

Accepted: 18 March 2015 Published online: 13 April 2015

\section{References}

1. Coussens LM, Werb Z. Inflammation and cancer. Nature. 2002;420:19-26.

2. Jemal A, Bray F, Center MM, Ferlay J, Ward E, Forman D. Global cancer statistics. CA Cancer J Clin. 2011;61:69-90.

3. Lutgens MW, Vleggaar FP, Schipper ME, Stokkers PC, van der Woude CJ, Hommes DW, et al. High frequency of early colorectal cancer in inflammatory bowel disease. Gut. 2008;57:1246-51.

4. Hussain SP, Hofseth LJ, Harris CC. Radical causes of cancer. Nat Rev Cancer. 2003;3:276-85

5. Uccelli A, Moretta L, Pistoia V. Mesenchymal stem cells in health and disease. Nat Rev Immunol. 2008;8:726-36.

6. Gonzalez MA, Gonzalez-Rey E, Rico L, Buscher D, Delgado M. Treatment of experimental arthritis by inducing immune tolerance with human adiposederived mesenchymal stem cells. Arthritis Rheum. 2009;60:1006-19.

7. lanni MD, Papa BD, loanni MD, Moretti L, Bonifacio E. Mesenchymal cells recruit and regulate T regulatory cells. Exp Hematol. 2008;36:309-18.

8. Ghannam S, Pene J, Moquet-Torcy G, Jorgensen C, Yssel H. Mesenchymal stem cells inhibit human Th17 cell differentiation and function and induce a T regulatory cell phenotype. J Immunol. 2010;185:302-12.

9. Fan H, Zhao G, Liu L, Liu F, Gong W, Liu X, et al. Pre-treatment with IL-1 beta enhances the efficacy of MSC transplantation in DSS-induced colitis. Cell Mol Immunol. 2012;9:473-81.

10. Sugai M, Aoki K, Osato M, Nambu Y, Ito K, Taketo MM, et al. Runx3 is required for full activation of regulatory $T$ cells to prevent colitis-associated tumor formation. J Immunol. 2011;186:6515-20.

11. Whiteside TL. Regulatory T, cell subsets in human cancer: are they regulating for or against tumor progression? Cancer Immunol Immunother. 2014;63:67-72

12. Takeda Y, Kaneda K, Jimma F, Shiobara N, Saniabadi AR, Wakabayashi I. Suppression of Th1 cytokine production by a peptide derived from C4b. Inflamm Res. 2013;62:951-9.

13. Brown JB, Cheresh P, Zhang Z, Ryu H, Managlia E, Barrett TA. P-selectin glycoprotein ligand-1 is needed for sequential recruitment of T-helper 1 (Th1) and local generation of Th17 T cells in dextran sodium sulfate (DSS) colitis. Inflamm Bowel Dis. 2012;18:323-32.

14. Fuss IJ, Heller F, Boirivant M, Leon F, Yoshida M, Fichtner-Feigl S, et al. Nonclassical CD1d-restricted NK T cells that produce IL-13 characterize an atypical Th2 response in ulcerative colitis. J Clin Invest. 2004;113:1490-7. 
15. Ito R, Kita M, Shin-Ya M, Kishida T, Urano A, Takada R, et al. Involvement of IL-17A in the pathogenesis of DSS-induced colitis in mice. Biochem Biophys Res Commun. 2008;377:12-6.

16. Chaudhry A, Rudra D, Treuting P, Samstein RM, Liang Y, Kas A, et al. CD4+ regulatory $T$ cells control $\mathrm{TH} 17$ responses in a Stat3-dependent manner. Science. 2009;326:986-91.

17. Koch MA, Tucker-Heard G, Perdue NR, Killebrew JR, Urdahl KB, Campbell DJ. The transcription factor T-bet controls regulatory $T$ cell homeostasis and function during type 1 inflammation. Nat Immunol. 2009;10:595-602.

18. Zheng Y, Chaudhry A, Kas A, deRoos P, Kim JM, Chu T, et al. Regulatory T-cell suppressor program co-opts transcription factor IRF4 to control $\mathrm{T}(\mathrm{H}) 2$ responses. Nature. 2009;458:351-6.

19. Takuji T, Kohno H, Suzuki R, Yamada Y. A novel inflammation-related mouse colon carcinogenesis model induced by azoxymethane and dextran sodium sulfate. Cancer Sci. 2003:94:965-73.

20. Lin X, Yi Z, Diao J, Shao M, Zhao L. ShaoYao decoction ameliorates colitisassociated colorectal cancer by downregulating proinflammatory cytokines and promoting epithelial-mesenchymal transition. J Transl Med. 2014;12:1-13.

21. Bak YK, Lampe JW, Sung MK. Effects of dietary supplementation of glucosamine sulfate on intestinal inflammation in a mouse model of experimental colitis. J Gastroenterol Hepatol. 2014;29:957-63.

22. Yang $Y$, Cai $X$, Yang J, Sun X, Hu C. Chemoprevention of dietary digitoflavone on colitis-associated colon tumorigenesis through inducing Nrf2 signaling pathway and inhibition of inflammation. Mol Cancer. 2014;13:1-14.

23. Liu L, Wang Y, Fan H, Zhao X, Liu D, Hu Y, et al. MicroRNA-181a regulates local immune balance by inhibiting proliferation and immunosuppressive properties of mesenchymal stem cells. Stem Cells. 2012;30:1756-70.

24. Liu L, Zhao G, Fan H, Zhao G. Mesenchymal stem cells ameliorate Th1induced pre-eclampsia-like symptoms in mice via the suppression of TNF-a expression. PLoS One. 2014;9, e88036.

25. Zhao X, Liu L, Liu D, Fan H, Wang Y, Hu Y, et al. Progesterone enhances immunoregulatory activity of human mesenchymal stem cells via PGE2 and IL-6. Am J Reprod Immunol. 2012;68:290-300.

26. Djouad F, Plence P, Bony C, Tropel P, Apparailly F, Sany J, et al. Immunosuppressive effect of mesenchymal stem cells favors tumor growth in allogeneic animals. Blood. 2003;102:3837-44.

27. El-Haibi CP, Bell GW, Zhang J, Collmann AY, Wood D, Scherber CM, et al. Critical role for lysyl oxidase in mesenchymal stem cell-driven breast cancer malignancy. Proc Natl Acad Sci U S A. 2012;109:17460-5.

28. Khakoo AY, Pati S, Anderson SA, Reid W, Elshal MF, Rovira II, et al. Human mesenchymal stem cells exert potent antitumorigenic effects in a model of Kaposi's sarcoma. J Exp Med. 2006;203:1235-47.

29. Qiao L, Xu Z, Zhao T, Zhao Z, Shi M, Zhao RC, et al. Suppression of tumorigenesis by human mesenchymal stem cells in a hepatoma model. Cell Res. 2008;18:500-7.

30. Greten FR, Eckmann L, Greten TF, Park JM, Li ZW, Egan LJ, et al. IKKbeta links inflammation and tumorigenesis in a mouse model of colitis-associated cancer. Cell. 2004;118:285-96.

31. Denning TL, Kim G, Kronenberg M. Cutting edge: CD4 + CD25+ regulatory T cells impaired for intestinal homing can prevent colitis. J Immunol. 2005;174:7487-91.

32. Fantini MC, Rizzo A, Fina D, Caruso R, Becker C, Neurath MF, et al. IL-21 regulates experimental colitis by modulating the balance between Treg and Th17 cells. Eur J Immunol. 2007:37:3155-63.

33. Huehn J, Polansky JK, Hamann A. Epigenetic control of FOXP3 expression: the key to a stable regulatory T-cell lineage? Nat Rev Immunol. 2009;9:83-9.

34. Fontenot JD, Rasmussen JP, Gavin MA, Rudensky AY. A function for interleukin 2 in Foxp3-expressing regulatory T cells. Nat Immunol. 2005;6:1142-51.

35. Liu Y, Zhang P, Li J, Kulkarni AB, Perruche S, Chen W. A critical function for TGF-beta signaling in the development of natural CD4 + CD25 + Foxp3+ regulatory T cells. Nat Immunol. 2008;9:632-40.

36. Sotiropoulou PA, Perez SA, Gritzapis AD, Baxevanis CN, Papamichail M. Interactions between human mesenchymal stem cells and natural killer cells. Stem Cells. 2006:24:74-85.

\section{Submit your next manuscript to BioMed Central and take full advantage of:}

- Convenient online submission

- Thorough peer review

- No space constraints or color figure charges

- Immediate publication on acceptance

- Inclusion in PubMed, CAS, Scopus and Google Scholar

- Research which is freely available for redistribution

Submit your manuscript at www.biomedcentral.com/submit 